Joanna Ciesielka

Université de Łódź

http://dx.doi.org/10.18778/8088-896-8.05

\title{
FAIRE APPRIVOISER L'AUTRE : SUR LES CAMPAGNES CONTRE L'HOMOPHOBIE EN POLOGNE, FRANCE ET ITALIE
}

«car en réalité l'homme est toujours aux côtés de son prochain »

(J. Tischner $\left.{ }^{1}\right)$

\section{À titre d'introduction}

Pour reprendre les paroles de Borrillo, l'homophobie, comme d'ailleurs la xénophobie ou le racisme, consiste à définir l'autre comme 'contraire', inférieur ou anomal ${ }^{2}$. Dans la société européenne, l'homophobie résulte de l'hétéronormativité qui « justifie culturellement la négation, le dénigrement et la stigmatisation de tous les comportements, toutes les idées, relations ou communautés qui ne se conforment pas précisément à la norme hétérosexuelle $\gg^{3}$. L'homophobie peut se traduire par les préjudices (l'attitude) ou la discrimination (le comportement $)^{4}$. Une de manières d'éveiller la réflexion de gens et d'influencer leur

1 J. Tischner, Myślenie wedtug wartości, Kraków, Znak, 1993, p. 186: „w rzeczywistości bowiem człowiek jest zawsze w pobliżu człowieka”, traduction vers le français J.C.

2 D. Borrillo, Omofobia. Storia e critica di un pregiudizio, Dedalo 2009, p. 7.

3 S. Mauceri, Omofobia come costruzione sociale. Processi generativi del pregiudizio in età adolescenziale, Milano, FrancoAngeli 2015, p. 16 : « legittima culturalmente la negazione, la denigrazione e la stigmatizzazione di ogni forma di comportamento, identità, relazione o comunità che non si conformi precisamente alla norma eterosessuale $\gg$; traduction de l'italien J.C.

4 Ibidem, p. $17:$ : Il pregiudizio [...] si manifesta laddove le credenze, le emozioni e le disposizioni ad agire di un individuo siano caratterizzate da ostilità nei confronti di coloro che si ritiene possano essere attratti, senza per questo tradursi in forme di azione manifesta contro gay. La discriminazione [...] la messa in atto di forme di azione locutorie e illocutorie tese a escludere, offendere, umiliare e/o ledere la forma psico-fisica di gay e lesbiche $\gg$. 
comportement est le recours à la publicité sociétale qui exprime et promeut les valeurs positives, humanitaires, les valeurs de solidarité et de civilité. Elle se sert de différentes 'techniques' et transmet ses messages en prenant diverses formes. Gadotti et Bernocchi en distinguent huit: sentimentale, pathétique, émouvante; dramatique, violente, choquante; agressive, accusatoire; rassurante, positive; humoristique, ironique; paternaliste, prescriptive; provocatrice, irrespectueuse, transgressive; informative, descriptive ${ }^{5}$.

L'objectif de cet article est de présenter une analyse qualitative des publicités de sensibilisation au problème de l'homophobie lancées au XXI siècle dans les trois pays : Pologne, France et Italie. Notre étude a été effectuée à la base du corpus de 17 affiches polonaises, 30 affiches françaises et 50 affiches italiennes. Il importe encore de préciser que nous n'avons pas pris en considération les campagnes concernant la légalisation des unions du même sexe.

\section{Pologne : cadre juridique et social}

La Constitution de la République polonaise de 1997 interdit toute sorte de discrimination d'une manière très générale: l'article 32 précise à ce propos seulement quelques domaines prévus par ladite loi comme « la vie politique, économique et sociale $\gg$. Dans un autre document, le Code du travail, on exprime explicitement l'interdiction de la discrimination fondée sur l'orientation sexuelle. Pour compléter ce cadre, il nous semble nécessaire d'ajouter que dans la législation polonaise il n'existe aucune forme juridique qui reconnaisse au nom de la loi les relations entre les personnes du même sexe.

Cet état de choses refléterait l'attitude des Polonais à l'égard des droits des gays et des lesbiennes, ainsi qu' à l'égard des unions civiles, ce qui sera confirmé par les données obtenues suite aux recherches effectuées en $2013^{6}$. Selon les résultats de l'enquête en question, presque deux tiers des Polonais sont d'avis que les couples homosexuels ne devraient pas avoir le droit de montrer leur mode de vie, plus que de deux tiers des interrogés n'acceptent pas le mariage entre les personnes du même sexe et plus de $80 \%$ de la société se prononcent contre la possibilité d'adopter les enfants par les homosexuels. Il est intéressant que seulement un tiers de la population de la Pologne est pour la possibilité d'établir des unions civiles entre les personnes du même sexe, tandis que $82 \%$ de gens se prononcent en faveur du droit permettant aux personnes qui restent dans une union durable de prendre des décisions liées aux funérailles du partenaire et reconnaissent leur

5 G. Gadotti, R. Bernocchi, La pubblicità sociale, Roma, Carocci, 2010.

6 Centrum Badania Opinii Społecznej, Raport z badania BS/24/2013 Stosunek do praw gejów i lesbijek oraz związków partnerskich, www.cbos.pl (page consultée le 20 octobre 2016). 
droit d'obtenir les informations sur la santé de l'ami(e) hospitalisé(e). Depuis cette perspective, il serait intéressant d'analyser quelques campagnes sociétales contre l'homophobie_sur la Vistule.

\section{Les campagnes polonaises}

En Pologne, la première campagne sociétale contre l'homophobie a eu lieu en 2003. Dans les rues des plus grandes villes polonaises sont apparus les panneaux qui représentaient des couples de gays ou de lesbiennes souriants avec un paysage urbain d'hiver au fond et l'inscription Niech nas zobacza ('Qu' ils nous voient'). L'objectif de cette campagne lancée par l'organisation non-gouvernementale $\mathrm{KPH}^{7}$ était de sensibiliser la société à la présence des personnes homosexuelles, de les faire sortir de l'ombre à la lumière du jour.

Quatre ans plus tard, à Varsovie, la même organisation a préparé la campagne Homofobia - tak to wyglada ('L'homophobie est comme ça'). Sur le fond noir il n'y avait que de simples inscriptions sous forme de questions: Co się gapisz lesbo? Co się gapisz pedale? ('Qu'est-ce que tu as à regarder, sale gouinasse ?', 'Qu'est-ce que tu as à regarder, pédé ?') sans aucune information supplémentaire. Après quelques jours, à la place de ces panneaux et posters on a accroché des photos d'une femme et d'un homme et les inscriptions respectivement : Pedał!/ Lesba! Słyszę to codziennie. Nienawiść boli. ('Pédé !/Sale gouine ! Je l’entends dire tous les jours. La haine fait mal').

En 2013 a vu le jour la campagne intitulée: Rodzice, odważcie się mówić ('Parents, ayez le courage de parler'), dont les destinataires et protagonistes étaient les parents des personnes homosexuelles et bisexuelles. Trois genres de slogans accompagnaient les photos qui montraient un ou deux parent(s) avec son fils ou sa/ leur fille sur le fond blanc: Córka nauczyła mnie odwagi. Elżbieta, matka lesbijki ('Ma fille m'a enseigné à être courageuse. Elżbieta, mère d'une lesbienne') ; Syn nauczył mnie jak ważne jest być sobą. Władysław, ojciec geja ('J’ai appris de mon fils comme il est important d'être soi-même. Władysław, père d'un gay') ; Córka nauczyła nas mówić otwarcie. Aneta i Rafał, rodzice lesbijki ('Notre fille nous a enseigné à parler ouvertement. Aneta i Rafal, parents d'une lesbienne'). Un acteur polonais Władysław Kowalski et son fils Jakub ont participé à cette campagne. Les panneaux et les posters ont été affichés en cinq grandes villes polonaises. Le maire de la ville de Varsovie Hanna Gronkiewicz-Waltz, le vice-président de la Diète et le délégué du gouvernement pour l'égalité des chances Agnieszka Kozłowska-Rajewicz ont accordé leur parrainage à cette campagne.

$7 \mathrm{KPH}$ - pol. Kampania przeciw Homofobii, une organisation non-gouvernementale polonaise dont l'objectif est, entre autres, de lutter contre la discrimination des personnes LGBT. 
La dernière campagne polonaise date du 2014 et s'intitule Ramię w ramię po równość 'Côte à côte pour l'égalité. Bien différente des campagnes organisées auparavant, elle ne consistait pas en la présentation des panneaux ou des affiches toutes prêtes dans la presse ou dans l'espace public. Bien que les auteurs de cette campagne aient préparé les panneaux avec quelques différentes inscriptions, tous ces textes étaient écrits suivant le même schéma : Jestem sojuszniczką/ sojusznikiem osób LGBT, bo... ce que l'on pourrait traduire en français comme 'Je suis un/e allié(e) des personnes LGBT parce que .... Les propositions finissaient par les subordonnées comme : miłość jest dla każdego, bez względu na orientację seksualną ('l'amour est pour tous quelle que soit son orientation sexuelle'); nie zgadzam się na przemoc i dyskryminację ('je suis contre la violence et la discrimination') ; chcę żyć w kraju, w którym moi homoseksualni przyjaciele nie są dyskryminowani ("je veux vivre dans un pays dans lequel mes amis homosexuels ne seront pas discriminés') ; różnorodność wzbogaca, a nie zagraża ('la diversité enrichit et ne menace point').

Plusieurs personnes du monde artistique, sportif et du show-business se sont photographiées en tenant lesdites inscriptions entre les mains, les organisateurs de la campagne ont donc fait recours aux arguments ad autoritatem en espérant que les Polonais suivraient le comportement de leurs idoles. Bien qu'elle ne le fasse pas expressis verbis, cette campagne se concentre non seulement sur l'acceptation des personnes homosexuelles, mais aussi sur l'égalité des droits de tous indépendamment de leur inclination sexuelle.

Toutes les campagnes sociétales décrites ci-dessus ont été lancées par les organisations non-gouvernementales. Il faudrait noter ici que les premières 'publicités' étaient privées de mots : seules les photos avaient pour objectif d'habituer les gens à la présence des personnes homosexuelles et de convaincre la société qu'elles ne sont pas différentes des hétérosexuels. La deuxième fait recours à l'émotivité du récepteur : la haine fait mal. Dans la troisième, adressée aux parents des gays et des lesbiennes, on invertit la division 'classique' des rôles : celle des parents qui enseignent et les enfants qui apprennent. Les enfants homosexuels démontrent à leurs parents l'importance des comportements francs, courageux, tout en attirant l'attention sur le respect mutuel.

Il serait intéressant d'aborder ne serait-ce que furtivement l'aspect linguistique de ces campagnes. En ce qui concerne les registres de langue utilisés dans les campagnes polonaises, on constate l'omniprésence d'un seul: registre standard, exceptions faites des mots vulgaires désignant les hommes et femmes homosexuels: pedał 'pédé' et lesba 'sale gouine'. Sans aucun doute, le type des messages transmis et leur contenu sont étroitement liés à la société polonaise, se voulant traditionnelle et au sein de laquelle on observe une très forte présence de l'Église catholique. Les publicités présentées ci-dessus s'inscrivent dans la stratégie de la lutte des personnes homosexuelles pour leurs droits appelée technique « à petits pas ». La première étape consisterait à montrer l'homosexualité 
en tant qu'orientation sexuelle sur le pied d'égalité avec l'hétérosexualité et à la présentation positive des homosexuels dans les médias. La deuxième aboutirait à la légalisation des unions civiles et la troisième aux mariages et à l'adoption des enfants par les gays ${ }^{8}$.

\section{France: cadre juridique et social}

En France, dans la loi $n^{\circ}$ 2004-1486 du 30 décembre 2004, qui instaurait la Haute autorité de lutte contre les discriminations et pour l'égalité, on a fait référence, de manière explicite, à l'orientation sexuelle: « seront pénalisées de façon quasi identique les provocations à la haine ou à la discrimination, l'injure ou la diffamation, concernant le racisme, l'homophobie, le sexisme, et l'handiphobie... » Il peut sembler surprenant qu'une telle mention ait été introduite dans une loi dans le pays où depuis 1999 il est légitime d'enregistrer le partenariat des personnes du même sexe $e^{9}$. On pourrait penser que l'ordre de l'instauration de ces deux lois aurait pu être différent et que dans un pays où existe le PACS, il n'y a pas de problèmes de discrimination basée sur l'orientation sexuelle, ce qui, comme le démontre le fragment cité ci-dessus, n'est pas forcément le cas. Il est intéressant de remarquer qu'une dizaine d'années plus tard, la législation française est allée encore plus loin. En effet, la loi n 2013-404 du 17 mai 2013 prévoit l'ouverture du législateur pour le mariage de couples de personnes du même sexe. Toutefois, malgré ces mesures législatives visant une vraie égalité des droits, les comportements LGBTphobes sont toujours présents au sein de la société française. Selon Le rapport sur l'homophobie 2016 publié par l'association SOS homophobie, les témoignages concernant les actes homophobes ont atteint leur pic en 2013 (avec 3517 de cas) pour diminuer au nombre de 1318 en $2015^{10}$.

\section{Les campagnes françaises}

Dans ce contexte, semble donc évidente l'organisation de diverses campagnes publicitaires dont l'objectif était de lutter contre l'homophobie ou de la prévenir. En décembre 2015, le ministère de l'Éducation nationale, de l'Enseignement supérieur et de la Recherche en ont lancé une dans les collèges, les lycées

${ }^{8}$ M. Grabowski, « Świat czy światy osób ze środowiska LGBT? Obraz własnego świata wartości i obraz świata społecznego w wynikach badania „fokusowego” » [in] I. Krzemiński (éd.), Naznaczeni. Mniejszości seksualne w Polsce - raport 2008, Warszawa, 2009, p. 130-148.

9 Article 515-1 du Code civil.

${ }^{10}$ Rapport sur l'homophobie 2016, Paris, SOS Homophobie, p. 13. 
et les universités pour sensibiliser les élèves et les étudiants aux discriminations de caractère homophobe. Les posters qui devaient être affichés dans les établissements éducatifs représentaient un/une jeune sur un fond noir. Ladite personne était entourée de propos prononcés souvent par les homophobes, par les ignorant(e)s qui n'avaient jamais rencontré un gay ou une lesbienne ou par ceux qui, tout simplement, ne savent rien sur l'homosexualité. Parmi les assertions et les questions représentées sur l'affiche, on lisait par exemple: « Comment tu sais que tu es lesbienne si t'as jamais couché avec un garçon ? », « C'est pas un truc de pédé », «Tu trouves pas qu'il y en a trop ? » et d'autres. En bas de l'affiche, dans un encadré orange, on voyait le slogan: « La violence des mots n'est pas plus acceptable que la violence physique. Lutter contre l'homophobie: le combat de toutes et de tous $\gg$.

Parmi les exemples concernant la lutte contre l'homophobie menée dans les institutions de l'enseignement supérieur, il faudrait mentionner encore un. Les posters de la campagne en question représentaient trois situations. Sur chacun, on voyait deux personnes qui se regardaient droit dans les yeux et qui étaient sur le point de s'embrasser. La première affiche montrait un garçon et une fille. Sous la photographie, on avait placé l'inscription: « Voici un couple hétérosexuel. Vous trouvez ça normal! » Deux autres affiches représentaient, respectivement, deux filles et deux garçons. Le message accompagnant ces photographies était le suivant: «Et là ? L'homosexualité n'est pas une erreur, le penser en est une $\gg$. Dans la partie inférieure de tous les trois posters se trouvait un encadré blanc dans lequel on pouvait lire la constatation: « L'homophobie mène à l'exclusion et au rejet. Les actes et les comportements homophobes peuvent avoir des conséquences désastreuses pour ceux qui en sont victimes $\gg$. Cette phrase qui ressemble à celles que l'on voit sur les paquets de cigarettes qui informent sur la gravité du tabagisme, était accompagnée d'incitation à affronter ce problème : « Luttons ensemble contre l'homophobie à l'université $\gg$.

Outre à être lancées par les autorités françaises, les campagnes contre l'exclusion et le rejet des personnes homosexuelles sont préparées et promues par les organisations non-gouvernementales. Dans ce contexte on ne pourrait pas passer sous silence une campagne particulièrement remarquable qui a été lancée par le Collectif contre l'Homophobie. Sur une des affiches on montre une jeune fille assise dans une salle de cours: on la voit de dos, elle y est toute seule et elle s'accoude sur un banc en couvrant son visage de mains. Deux mots sont écrits au tableau en grands caractères rouges: sale gouine. Dans la partie supérieure du poster, sur un fond noir, le lecteur est informé sur la situation de la jeune fille: « Jeanne, 18 ans. Bonne élève, admise en classe prépa mais exclue de la sienne $\gg$. Dans la partie inférieure de la photographie, les auteurs de la campagne nous informent que l'homophobie exclut. Le message transmis par l'autre affiche, dont l'esthétique est semblable 
à celle de la précédente, est beaucoup plus fort. La photographie représente une pierre tombale avec l'inscription suivante: «A notre fils aimé Manuel 1989-2008 », dans sa partie inférieure on voit une autre inscription : «L'homophobie tue $\gg$. Au-dessus de la photographie, on peut lire quelques informations sur Manuel, à savoir: «Manuel, 19 ans, homosexuel. Ses parents l'aimaient, ses amis l'aimaient, tout le monde l'aimait. Enfin, presque tout le monde $\gg$. Le mot presque, paraît, dans le contexte de l'image, un des motsclés du message transmis soulignant l'importance de l'acceptation des jeunes de la part de leur entourage, car les conséquences d'une attitude contraire peuvent être tragiques et irrémédiables.

Il serait important de signaler qu'en France les campagnes contre l'homophobie concernent aussi la discrimination au travail (par exemple, celle menée par CFDT Pays de la Loire et Parti Socialiste) ou dans le monde du sport.

Pour ce qui est de l'aspect linguistique des campagnes françaises, il faudrait remarquer l'usage des mots comme homophobe et homophobie, ce qui n'était pas le cas dans les campagnes polonaises. Les registres de langue utilisés sont deux: le français standard et le français familier. Le premier domine dans les slogans et dans les publicités adressées aux adultes, le deuxième apparaît dans les messages qui rapportent les phrases prononcés par les jeunes et dont ils sont les destinataires. Les auteurs des campagnes françaises font souvent appel à la solidarité des membres de la société, en les invitant à agir ensemble, ce que l'on observe à travers l'usage de la première personne du pluriel à l'impératif.

\section{Italie : cadre juridique et social}

La Constitution de la République italienne de 1997 interdit toute sorte de discrimination d'une manière très générale, ce qui devrait garantir à tous, les minorités sexuelles inclues, une égalité de chances, néanmoins, dans la réalité, ceci n'est pas toujours le cas. Ce n'est qu'en 2003 et en 2008, que la directive du Conseil 2000/78/CE contribuant à la création d'un cadre général en faveur de l'égalité de traitement en matière d'emploi et de travail a été transposée en droit italien.

Le 5 juin 2016 est entrée en vigueur la loi sur les unions civiles appelée aussi la loi Cirinnà (du nom du sénateur Monica Cirinnà qui l’a proposée).

Pourtant, en ce qui concerne les comportements homophobes, selon le sondage de l'ISTAT de 2012, presque $40 \%$ des personnes qui se sont déclarées gays ou bisexuelles, ont été victimes de discrimination dans le milieu scolaire ou de travail ${ }^{11}$.

11 S. Mauceri, op. cit., p. 11. 
Joanna Ciesielka

\section{Les campagnes italiennes}

En Italie, dans le laps de temps étudié, nous pouvons observer la participation des autorités gouvernementales et régionales (au moins de certaines régions) dans les campagnes de sensibilisation contre l'homophobie. Présentons-en une comme exemple. Elle a été organisée en 2009 par le Ministère pour l'Égalité des chances (it. Dipartimento per le Pari Opportunità) et elle a été composée de spots publicitaires et panneaux. Sur les affiches de couleur noire, dans la partie supérieure à gauche, on a représenté un encadré ressemblant à un bulletin de vote, sur lequel on a mis trois mots: omosessuale, eterosessuale et non importa (fr. homosexuel, hétérosexuel et cela n'a pas d'importance) avec une case cochée en rouge avant la dernière option. A droite, dans la partie centrale et inférieure, on voit l'inscription: Nella vita certe differenze non possono contare. Rifiuta l'omofobia ce que l'on pourrait traduire en français comme 'Dans la vie, certaines différences ne peuvent pas être importantes. Rejette l'homophobie'.

Un autre panneau intéressant, cette fois-ci proposé par les autorités de la ville de Rome, a vu le jour en 2003. Dans la partie gauche de l'affiche on a représenté le visage d'un jeune homme avec l'empreinte rouge du sceau Eterosessuale sur le front. Dans la partie droite, au milieu une question nous incite à la réflexion: Cosa guardi in una persona? ('Qu'est-ce que tu regardes dans une personne ?'), dans la partie inférieure du même côté l'inscription informe le récepteur que Eterosessuali. Gay. Lesbiche. Transsessuali. Le diversità sono normali, i pregiudizi no ('Les différences sont normales, les préjugés non').

Beaucoup de campagnes italiennes ont été lancées par les organisations non-gouvernementales, comme Arcigay, Arcilesbica, Arci et d'autres. En 2009, deux députés italiens: Anna Paola Concia et Jean Leonard Touadì ont pris part dans une campagne contre les discriminations et l'homophobie. L'affiche de cette campagne représentait lesdits députés, l'une en face de l'autre, avec les poitrines nues et les bras croisés. Le message suivant, qui fait référence aux images stéréotypées, accompagnait la photographie: Ci chiami sporco negro e lesbica schifosa, ma ti offendi se ti chiamano italiano mafioso ce que l'on pourrait traduire en français comme 'Tu nous appelles sale nègre et lesbienne dégoûtante, mais tu te vexes si on t'appelle mafioso italien'. En dessous on a mis deux phrases qui constituaient un commentaire à la phrase précédente: Il razzismo è come un boomerang. Prima o poi ti ritorna ('Le racisme est comme un boomerang. Tôt ou tard, il te reviendra').

Une autre campagne qui devrait être mentionnée ici se rapporte à la longévité du couple, ce qui évoque, sans aucun doute, les valeurs familiales. Sur les panneaux, qui avaient pour objectif de convaincre la société d'introduire les mêmes droits pour tous, on voyait deux femmes et l'inscription : Agata e Angela da Siracusa da 27 anni insieme con amore contro l'odio e le difficoltà. L'amore vince sempre 
sull'odio. Una legge contro l'omofobia anche in Italia. Subito! ('Agata et Angela de Syracuse depuis 27 ans ensemble avec amour face à la haine et aux difficultés. L'amour vainc toujours la haine. Une loi contre l'homophobie en Italie aussi. Tout de suite!') Il existe, bien sûr, la version «masculine» de ce panneau, représentant un couple de gays qui vivent ensemble depuis 45 ans. Il faudrait noter ici que les posters de cette campagne ont été préparés à l'occasion de la Journée internationale contre l'homophobie que l'on célèbre chaque année le 17 mai. D'ailleurs, il y en a beaucoup d'autres à promouvoir cet événement, toutefois, il semble nécessaire de mentionner un panneau grâce à son caractère accrocheur, selon certains, provocateur et même outrageant. Sur un fond blanc, dans la partie droite de l'affiche, sur un quart du poster, on a présenté un fragment de La Pietà de Michelange et sur les trois quarts, on voit l'inscription suivante : L'omotransfobia è odio che ti uccide. Non esserne complice ('L'homotransphobie est la haine qui te tue. Ne soit pas son complice'). Les couleurs contrastées de la police (le rose - du premier et du dernier mot, le noir - le reste du message) attirent l'attention du récepteur qui ne peut pas passer indifférent devant une telle affiche. De plus, la création du message de manière à pouvoir l'interpréter à différents niveaux, a provoqué des discussions enflammées, grâce auxquelles l'opinion publique est devenue peut-être plus sensibilisée au problème de l'homotransphobie.

En ce qui concerne l'aspect linguistique des campagnes italiennes étudiées, on y met en opposition, d'un côté : l'homophobie, traitée comme un préjudice et une cause de souffrances et, de l'autre, les orientations sexuelles (hétéro-, homo-, bi- et trans-) considérées comme un simple signe de différence et non d'une anormalité. On peut l'apercevoir au niveau lexical, à travers les mots utilisés: omofobo, omofobia, pregiudizio, diversità, diverso, normale. Dans les phrases impératives, on observe avant tout l'usage de la deuxième personne du singulier, ce qui relève sans doute du caractère que l'on pourrait appeler 'direct' de la langue italienne.

\section{Conclusion}

Au préalable, il faut souligner que la comparaison quantitative des compagnes contre l'homophobie n'a pas été une approche principale étant donné que le nombre de matériau variait considérablement suivant le pays étudié. De ce fait, dans la section consacrée à la Pologne, nous avons recensé toutes les campagnes qui ont été beaucoup moins nombreuses par rapport aux autres pays évoqués.

En ce qui concerne l'analyse qualitative, nous sommes capables d'en distinguer les différences manifestes. Par exemple, la langue utilisée dans les campagnes polonaises diffère visiblement de celles menées en France ou en Italie. Contrairement à Rome et à Paris, dans les campagnes faites au bord de la Vistule on remarque tout de suite que leurs auteurs semblent peu enclins à appeler les choses par leurs noms - même pas une seule fois le mot 'homophobe' ou 'homopho- 
bie' n'apparaissent - uniquement dans une campagne on a eu recours à l'émotion et à l'empathie du destinataire. En revanche, dans les campagnes françaises le langage est conforme au public auquel elles s'adressent : d'où les éléments de la langue populaire et du verlan qui y sont présents.

Les Français n'hésitent pas à avoir recours à des images assez fortes (comme la pub représentant le tombeau) et ne reculent pas devant l'utilisation des mots du type 'homophobie'. Dans les campagnes françaises et italiennes le soi-disant 'ennemi' se trouve plutôt dans l'environnement extérieur (école, université, travail, associations sportives) tandis qu'en Pologne on n'oublie pas la famille. En France et en Italie on tente, d'un côté, de sensibiliser au problème de l'homophobie et du rejet et, de l'autre, de proposer une aide (asiles) aux personnes touchées par l'exclusion sociale à cause de ses inclinations sexuelles. Les campagnes italiennes semblent plus variées et plus intéressantes depuis la perspective visuelle (le cadre du présent article ne permet pas pourtant pas d'aborder cette question en profondeur). Ces campagnes se réfèrent ouvertement aux valeurs de famille montrant les relations homosexuelles comme autant durables que celles des hétérosexuels. C'est ainsi qu'on tente de briser les stéréotypes sur les personnes homosexuelles. Dans les campagnes italiennes on utilise les procédés « made in Italy » (vulgarisation de l'ouverture d'esprit comme « prodotto tipicamente friulano »), on évoque le non-hétérosexualité comme une différence qui enrichit et ne divise pas les gens.

Il est opportun de noter ici qu'en France et en Italie certaines campagnes sont soutenues par les autorités régionales ou nationales, ce qui n'est pas le cas en Pologne surtout ces dernières années. Le présent article n'est qu'une introduction à la problématique qui, nous l'espérons, incitera à des études ultérieures en la matière.

\section{Taming the 'odd' ones, a comparison of anti-homophobia campaigns in Poland, France, and Italy}

The objective of the article is to present as well as compare $21^{\text {st }}$ century anti-homophobia campaigns in Poland, France, and Italy. The information found in the printed press was used as the research material. The author presents legal situations of the LGBT communities in the aforementioned countries and moves on to analyzing lexical and visual means used in the campaigns that attempt to persuade society that the 'odd' ones (mostly homosexuals, lesbians, bisexuals, and transsexuals) are deserving of respect and equal treatment. Materials that concerned different forms of legalization of single-sex relationships were not the subject of this analysis.

Keywords: anti-homophobia - campaigns - Poland - France - Italy

Mots-clés : publicité sociétale - homophobie - Pologne - France - Italie 